CERN LIBRARIES, GENEVA

CM-P00062404

FROM LATTICE QCD TO NUCLEAR PHYSICS

\author{
H.J. Pirner \\ CERN - Geneva
}

Invited Talk for the

1987 Winter School at

Les Houches

CERN-TH.4727/87

May 1987 


\section{FROM LATTICE QCD TO NUCLEAR PHYSICS}

Hans J. Pirner

CERN, Geneva, Switzerland

\section{GENERAL INTRODUCTION}

Perturbative quantum chromodynamics (OCD) has been extensively studied and agrees well with experiment. As an example, let us look at the angular distribution of two-jet events measured in the UA 1 experiment at CERN (see Fig. 1). The experimental data [1] follow the $1 /(1-\cos \theta)^{2}$ behaviour of Rutherford scattering. Even small visible corrections from scaling violations $\left(Q^{2}\right.$-dependence) in the structure functions are calculable. The gluon exchange manifests itself in the same way as photon exchange in electrodynamics. However perturbative $\mathrm{OCD}$ only becomes applicable at length scales $1 / \mathrm{Q}^{2} \leqslant(0.1 \mathrm{fm})^{2}$. The interesting physics of interacting hadrons and nuclei, is given by a scale of $1 \mathrm{fm}$ to $10 \mathrm{fm}$. It therefore needs non-perturbative methods to tackle the QCD Lagrangian. Various approaches have been proposed. Since an expansion in the strong coupling constant $\alpha_{s}\left(Q^{2}\right)$ of $Q C D$ is not possible, an expansion in $1 / N_{c}$ has been suggested, where $N_{c}$ is the number of colours [2,3]. One obtains a theory of weakly interacting mesons with couplings $g_{M}=O\left(1 / N_{c}\right)$ and masses $O(1)$. Baryonic solutions have masses $O\left(1 / g_{M}\right)=O\left(N_{c}\right)$, which possibly indicates that they are soliton solutions of the meson-field equations. The existence of a gluon master field proposed by Witten [4] in the $\mathrm{N}_{c} \rightarrow \infty$ limit would allow quantitative calculations, but up to now no results in four dimensions are available. There are calculations trying to relate the $Q C D$ Lagrangian to a Skyrme-type effective action assuming chiral symmetry breaking [5]. But most of the progress in this field has been made in applications of Skyrme-type models [6].

A different mostly numerical approach to non-perturbative $Q C D$ has been followed during the last years, namely lattice $Q C D[$ [7]. By discretizing continuous space-time on a finite lattice one considers a

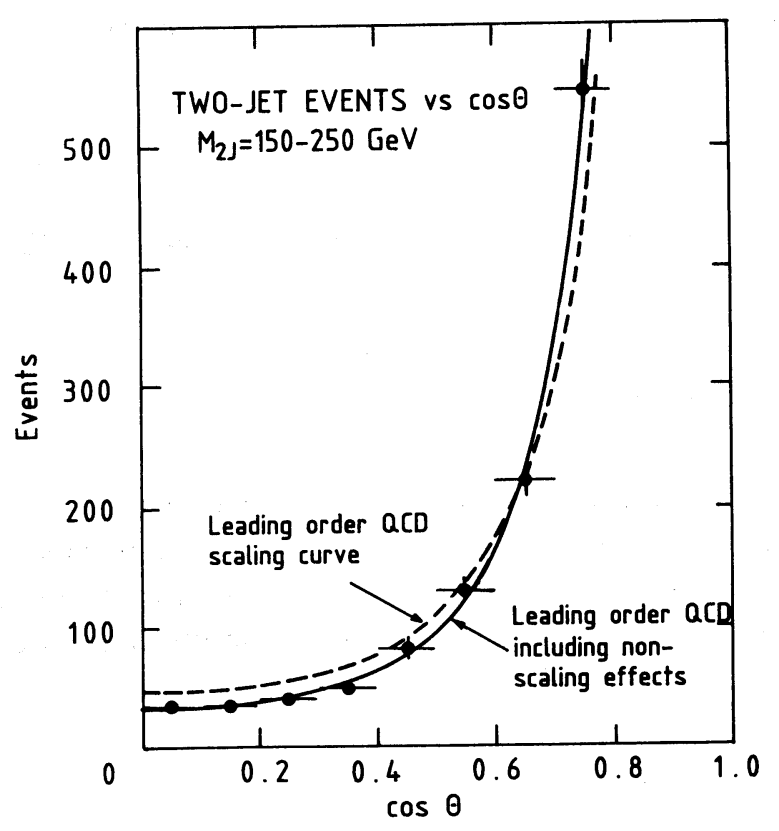

Fig. 1 Two jet events as a function of $\cos \theta$ from Ref. 1 . The $\mathrm{qq} \rightarrow \mathrm{qq}, \mathrm{gg} \rightarrow \mathrm{gg}$ and $\mathrm{gq} \rightarrow \mathrm{gq}$ processes give the Rutherford scattering distribution (dashed line) $d \sigma \propto 1 /(1-\cos \theta)^{2}$. The full line includes the scaling violations in the structure functions. 
finite number of gauge-field configurations, which can be simulated numerically. The finite lattice constant regularizes an otherwise diverging field theory. The lattice method has developed into a field where the computational possibilities play an important part in the discussion. However there are also serious, not yet understood, physics problems concerning the treatment of systems with finite fermion density [8].

The work I am going to describe here has involved a moderate numerical effort up to now. Its aim is primarily conceptual. Can we define an effective $Q C D$ theory, where the short-wavelength gluon degrees of freedom are eliminated in favour of a colour-neutral field characterizing the vacuum and giving confinement. The role of the polarizable medium is played by the high-frequency parts of the gauge fields. Since this effective theory has the same appearance as electrodynamics of dielectric media, it is called colour dielectrics.

\section{From the 'macroscopic' Hamiltonian}

$$
H=\int \sum_{i=1}^{8} 1 / \epsilon\left[\vec{D}_{i}^{2}+\vec{H}_{i}^{2}\right] d^{3} x
$$

it is easily seen that colour displacement fields $\vec{D}_{i}$ and magnetic fields $\vec{H}_{i}$ cannot subsist in a medium where $\epsilon=0$, because the energy associated with them would be infinite. The vacuum therefore has $\epsilon=0$. The dynamics of confinement is reduced to find the value of the dielectric field in every point in space around the colour charges. The region with $\epsilon=0$ forms a bag around the $\epsilon \neq 0$ region inside the hadron, where colour is present and not influenced by vacuum fluctuations.

My paper will consist of three parts. In Section 2 I give an introduction to the main physics of lattice gauge theory. Section 3 gives an outline of the colour dielectric model and first numerical results on the effective action after one block-spinning step. This part of the paper is based on joint work with $\mathrm{J}$. Wroldsen [9]. Section 4 is devoted to a report about phenomenological work, done in collaboration with G. Chanfray and A. Schuh, which uses the colour dielectric model as a starting point for calculations of the free nucleon and nuclear matter or finite nuclei.

\section{INTRODUCTION TO CONCEPTS OF LATTICE GAUGE THEORY}

For simplicity we are going to discuss non-Abelian SU(2) chromodynamics of gluon fields. That means the gluon fields have a $2 \times 2$ matrix representation by the Pauli matrices $\vec{\sigma}_{i}(i=1,3)$ as

$$
A_{\mu}(x)=1 / 2 \sum_{i=1}^{3} A_{\mu}^{i} \sigma^{j} .
$$

Having discretized the three space and one time dimensions we introduce as relevant degrees of freedom the link variables

$$
U_{\mu}(x)=\operatorname{expig} \int_{x}^{x+e_{\mu}} A_{\nu} d \xi_{\nu}=a_{0}+i \vec{\sigma} \vec{a}
$$

which symbolize the integral of the gauge field between a site $x$ of the lattice to the neighbouring site $\mathrm{x}+\mathrm{e}_{\mu}$, one lattice spacing away from $\mathrm{x}$ in the $\mu=(1,4)$ direction. This link variable has a $2 \times 2$ matrix representation $U_{\mu}=a_{0}+i \vec{\sigma} \vec{a}$ given by four numbers $\left(a_{0}, \vec{a}\right)$. It is unitary, which means that the product of a link in the positive $\mu$-direction with the same link traversed in the opposite $\mu$-direction gives the unity operator

$$
\mathrm{U}_{\mu}(\mathrm{x}) \mathrm{U}_{\mu}^{+}(\mathrm{x})=\mathrm{U}_{\mu}(\mathrm{x}) \mathrm{U}_{-\mu}\left(\mathrm{x}+\mathrm{e}_{\mu}\right)=1
$$

Unitarity restricts the four quantities $\left(a_{0}, \vec{a}\right)$ to the unit circle $a_{0}^{2}+\vec{a}^{2}=1$. The effect of a gauge transformation $G(x)$ on $U_{\mu}(x)$ can be seen by expanding $U_{\mu}$ for small gauge fields and using the standard continuum gauge transformation $A_{\mu}(x) \rightarrow A_{\mu}^{\prime}=(1 / i)\left[\partial_{\mu} G(x)\right] G^{-1}(x)+G(x) A_{\mu}(x) G^{-1}(x)$. We get 


$$
\begin{aligned}
& 1+i A_{\mu} d x_{\mu} \rightarrow \\
& 1+\left\{[G(x+d x)-G(x)] / d x_{\mu} G^{-1}(x)+i G(x) A_{\mu}(x) G^{-1}(x)\right\} d x_{\mu} \\
& \approx G(x+d x)\left(1+i A_{\mu} d x_{\mu}\right) G^{-1}(x)
\end{aligned}
$$

Therefore a link $U_{\mu}(x)$ transforms by applying the gauge transformations $G$ and $G^{-1}$ on the end points $\left(x+e_{\mu}\right)$ and $x$ of the link. Consequently, the natural choice for a gauge-invariant action is made from closed loops, the smallest of which are plaquettes with four links. Indeed this is the form of the Wegner-Wilson $[10,11]$ action $S(U)$ (see Fig. 2)

$$
\begin{aligned}
S(U) & =\sum_{\text {all plaquettes }}\left[1-1 / 2 \operatorname{tr}\left(U_{i \ell} U_{l k} U_{k j} U_{j i}\right)\right] \\
& =\sum S_{\square}
\end{aligned}
$$

The partition function is given by the sum over all possible link configurations weighted with the 'Boltzmann factor'

$$
Z=\int D U e^{-\beta S(U)}
$$

Here the integral can always be thought of as a multiple integration over the constrained vector variables $a_{0}, \vec{a}$ at all links, i.e.

$$
\int D U=\int \prod_{\text {links }} d a_{0, \ell} d \vec{a}_{\ell} \delta\left(a_{0, \ell}^{2}+\vec{a}_{\ell}^{2}-1\right)
$$

The OCD coupling constant $g$ enters into the expression $\beta=4 / \mathrm{g}^{2}$. In the strong coupling limit, i.e. for small $\beta$, we see that the exponential allows values of $S_{\square} \rightarrow 2$ which are related to strong fluctuations of the links, e.g.

$$
(\cup \cup \cup \cup)=\left(\begin{array}{cc}
-1 & 0 \\
0 & -1
\end{array}\right)
$$

For weak coupling or large $\beta$ the 'Boltzmann' weight prefers $S_{\square} \rightarrow 0$, i.e. trivial link variables near the identity, namely

$$
(\cup \cup \cup \cup)=\left(\begin{array}{ll}
1 & 0 \\
0 & 1
\end{array}\right)
$$

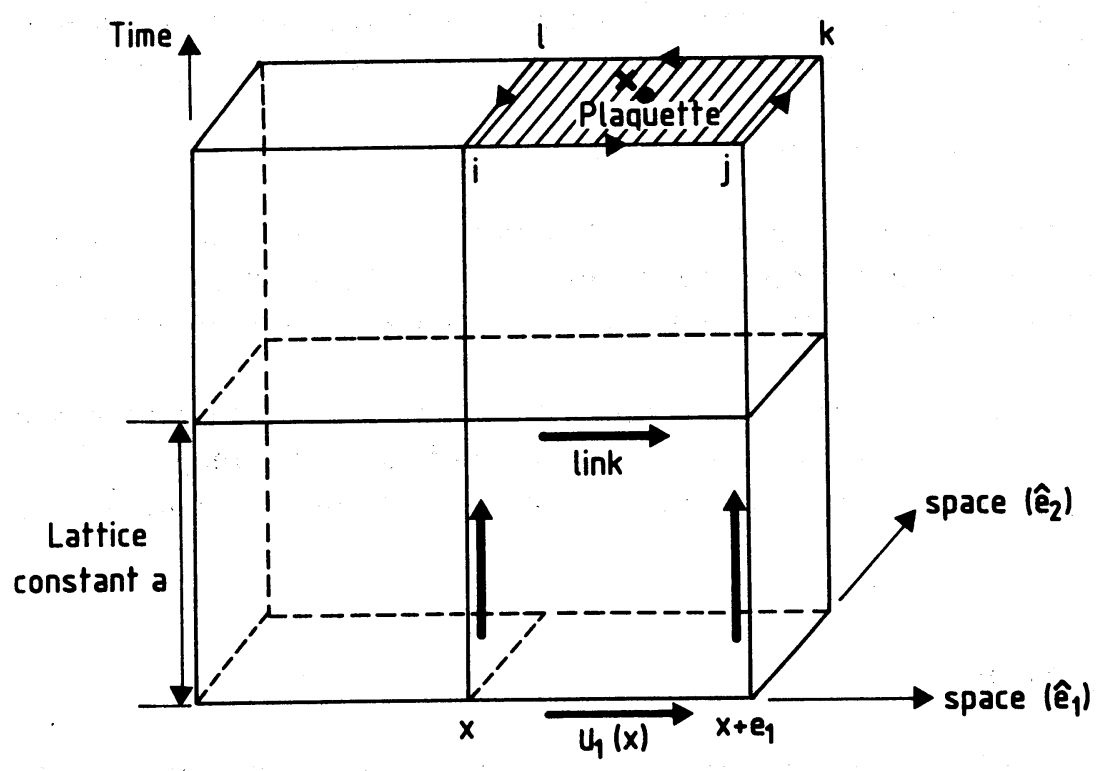

Fig. 2 Schematic picture of a lattice with the link variables and a basic plaquette. 
Part of the normal numerical problem is associated with the necessity of working in the weak-coupling domain, where the fluctuations of the gauge fields are suppressed and the 'thermalization' of the system takes very long times.

An important point concerns the continuum limit of the action given in Eq. (4), when the lattice spacing a goes to zero. Take a plaquette in the $\hat{e}_{1}, \hat{e}_{2}$ plane and expand all link variables around the centre of the plaquette; then one gets (Fig. 2).

$$
\begin{aligned}
S_{\square}=1-1 / 2 \operatorname{tr} & {\left[\exp i g \bar{A}_{1}\left(x-1 / 2 a \hat{e}_{2}\right) a\right.} \\
& \exp i g \bar{A}_{2}\left(x+1 / 2 a \hat{e}_{1}\right) a \\
& \exp -i g \bar{A}_{1}\left(x+1 / 2 a \hat{e}_{2}\right) a \\
& \left.\exp -i g \bar{A}_{2}\left(x-1 / 2 a \hat{e}_{1}\right) a\right]
\end{aligned}
$$

Let us use the Baker-Hausdorf formula $e^{x} e^{y}=e^{x+y+1 / 2[x, y]}$ to derive

$$
\begin{aligned}
S_{\square} & =1-1 / 2 \operatorname{tr} \exp \left\{i a^{2} g\left(\partial_{1} A_{2}-\partial_{2} A_{1}\right)-a^{2} g^{2}\left[A_{1}, A_{2}\right]\right\} \\
& =1-1 / 2 \operatorname{tr} \exp i a^{2} g F_{12}
\end{aligned}
$$

where the commutator term does not vanish, because of the non-Abelian nature of the gauge fields. For small lattice spacing $\mathrm{a}$, we can expand the exponential. The trace of the first-order term $\operatorname{tria}^{2} \mathrm{gF}_{12}$ vanishes when we integrate over all group elements, and the second-order term gives the desired continuum action for $\beta=4 / \mathrm{g}^{2}$ :

$$
\begin{aligned}
\beta \Sigma S_{p} & =1 / 8 \operatorname{tr} \beta g^{2} a^{4} \sum_{x} F_{\mu \nu}(x) F_{\mu \nu}(x)= \\
& =g^{2} \beta / 4 \cdot \int d^{4} x \operatorname{tr} 1 / 2 F_{\mu \nu} F_{\mu \nu}
\end{aligned}
$$

Asymptotic freedom can be expressed in this language as a relation between the OCD coupling constant $g$ and the lattice spacing $a$, which has the following form for SU(2)

$$
g^{2} / 4 \pi=3 \pi /\left[(11 / 2) \ln \left(1 / \Lambda^{2} a^{2}\right)\right]
$$

Here $\Lambda$ is the dimensional size parameter of SU(2) $Q C D$, i.e. $\Lambda_{O C D}$. In order to obtain the continuum limit one has to make the lattice spacing a sufficiently small, which makes $\mathrm{g}$ also small. Then one is faced with the problem of slowing down the equilibration of the system together with a real lattice size of small dimensions $(1-2 \mathrm{fm})$ [4]. Recently calculations with (24-32) ${ }^{4}$ lattices have been started. Turning the above formula $(10)$ around, a numerical simulation of a physical dimensional parameter $\mathrm{m}^{2}$ is considered as scaling when it behaves like the physical measurable QCD constant, namely as

$$
\mathrm{m}^{2}=\text { const. } \Lambda^{2}=\text { const. } 1 / \mathrm{a}^{2} \exp \left(-6 \pi^{2} \cdot \beta / 11\right) .
$$

Taking a numerical expectation value at several values of $\beta$, a comparison with Eq. (11) establishes whether one is close enough to the continuum limit. Clearly numerical lattice simulations can only give us physical quantities expressed in units of $\Lambda$, which has to be taken from experiment.

The numerical technique we use is the heat-bath method [12]. Working our way through the lattice we generate random four vectors $a_{0}, \vec{a}$ for each link according to the weight $e^{-\beta S(U)}$ which is determined by the neighbouring links. Once the system is in equilibrium we can evaluate any desired correlation function.

\section{COLOUR DIELECTRICS}

Colour dielectrics is based on regarding the $Q C D$ vacuum as a medium characterized by a dielectric field. It is a theoretical approach which promises to construct an effective action for a bag model directly from 
the OCD Lagrangian. Clearly for many experiments in nuclear physics at higher energies we need a theoretical description which at the same time contains the long-range features of confinement without giving up the elementary quark and gluon degrees of freedom. After all it is the search for these QCD degrees of freedom which motivates the experimental work. Studying the transition from asymptotically free $Q C D$ at short distances to confining hadron dynamics at long distances is the exciting task we are faced with. For this problem a transformation is ideal which maps a theory with a lattice spacing a into one with lattice spacing 2a. Since such a mapping or block-spin transformation can be carried out repeatedly we are led to an effective model Lagrangian for long-distance hadron physics. We define a chain of field theories at $a_{0}, 2 a_{0}, 4 a_{0}, \ldots, 2^{n} a_{0}$, then the continuum limit $a_{0} \rightarrow 0$ is achieved by letting $n \rightarrow \infty$ such that $\lim 2^{n} a_{0} \rightarrow$ finite.

In Nielsen and Patkos [13] a perturbative calculation of one block-spinning step towards the colour dielectric Lagrangian is made. Mack [14] introduces models of effective actions and a definition of the colour dielectric field, which is used in our numerical work. For a general discussion of the renormalization group method in Monte Carlo calculations we refer the reader to the article of Wilson [15].

Constructing the block variables for the effective action is a somewhat arbitrary process. We proceed in the following way. First we select every second hypercube on the lattice. Then we make gauge transformations on all sites of a chosen hypercube in order to make its link variables $\widetilde{U}$ close to the identity, i.e. $1 / 2 \operatorname{Tr} \widetilde{U} \approx 1$. We iterate this gauge transformation program approximately 5 to 9 times for each hypercube. Once this is done all the information is in the links $U_{i}$ which connect the individual hypercubes. In 4 dimensions there are 8 interconnecting links, the average of which defines the new link variables $V$ times the colour dielectric field $\chi$. This scalar field $\chi$ is necessary, otherwise the new link variables $V$ would not be unitary.

$$
\chi \cdot V=1 / 8 \sum_{\substack{i=1 \\ \text { connecting }}}^{8} U_{i}
$$

It can easily be shown that for unitary matrices $V=b_{0}+i \vec{\sigma} \cdot \vec{b}$ and $U_{i}=a_{0}^{i}+i \vec{\sigma} \cdot \vec{b}_{i}$ we get

$$
\operatorname{det}\left(\chi b_{0}+\chi \vec{i} \vec{\sigma} \vec{b}\right)=\chi^{2}\left(b_{0}^{2}+\vec{b}^{2}\right)=\chi^{2}
$$

and

$$
\begin{aligned}
& \operatorname{det}\left(1 / 8\left(\sum \mathrm{a}_{0}^{i}+i \vec{\sigma} \sum \vec{a}^{i}\right)\right)= \\
& =\left(1 / 8 \sum a_{0}^{i}\right)^{2}+\left(1 / 8 \sum \vec{a}^{i}\right)^{2} \leq \max _{v i}\left(a_{0}^{2}+\vec{a}_{i}^{2}\right)=1
\end{aligned}
$$

Therefore, without limiting our generality, $\chi$ will take values between 0 and 1 .

For the new effective action we assume the following form:

$$
\begin{aligned}
& e^{-S_{e f f}}(\chi \cdot V)= \\
& \exp \left[-\beta^{\prime} \sum_{\square}\left(1-1 / 2 \operatorname{tr}\left(\chi^{\vee}\right)\left(\chi^{\vee}\right)\left(\chi^{\vee}\right)\left(\chi^{\vee}\right)\right)-\sum_{x}\left(m^{2} \chi^{2}+\lambda \chi^{4}\right)\right]
\end{aligned}
$$

The new plaquettes are now of length $2 a$, and if it is natural to decorate each new link $V$ with the colour dielectric field $\chi, 0 \leq \chi \leq 1$. That is how we averaged the interconnecting gauge configurations anyway. In the continuum limit the above action will give us the desired result of a dielectric theory,

$$
\begin{aligned}
L & =1 / 2 \operatorname{tr} B_{\mu \nu} B_{\mu \nu} \chi^{4}(x)+L(x)= \\
& =1 / 2 \sum_{i=1}^{3}\left(\vec{E}_{i}^{2}-\vec{B}_{i}^{2}\right) \cdot \epsilon+L(x) .
\end{aligned}
$$


where

$$
\epsilon=\chi^{4}
$$

i.e. the colour dielectric constant $\epsilon(x)=\chi^{4}(x)$ modifies the electric and magnetic field strengths $\vec{E}_{i}^{2}$ and $\vec{B}_{i}^{2}$. In simulations on an $8^{4}$ lattice using $\sim 600$ sweeps through the lattice, we obtained preliminary results (Fig. 3) for the dielectric constant $\langle\epsilon\rangle=\left\langle\chi^{4}\right\rangle$. For strong coupling $\epsilon$ approaches zero, whereas, at $\beta=2, \epsilon$ increases drastically. From simulations of the string tension it is well known that at $\beta=2$ a cross-over transition takes place, which is reflected in the behaviour of $\epsilon$. Using Schwinger-Dyson [16] equations, we derive the new effective couplings $\beta^{\prime}, \mathrm{m}^{2}$, and $\lambda$. The self interaction of the colour dielectric field is shown in Fig. 4. It documents the tendency for the effective action to assume its minimum near $\chi=0$ when the coupling $\mathrm{g}$ is large. This supports the basic hypothesis of the dielectric theory of confinement. Colour sources are the source of electric inductions $\vec{D}_{i}$, which are related to the colour electric fields $\vec{E}_{i}$ by $\vec{D}_{i}=\chi^{4} \vec{E}_{i}$. Therefore, the $\vec{D}_{i}$ fields can only be non-zero where $\epsilon \neq 0$. If $\epsilon=0$ is the unique classical vacuum then $\chi \neq 0$ costs energy and this prevents the $\vec{D}$ fields from spreading. As a result, a flux tube will form between quarks of opposite charges in the region where $\epsilon \neq 0$ only. Colour is confined. In the next section we will see some phenomenological applications of this mechanism. The numerical calculations [9] confirm the preliminary observed trends.

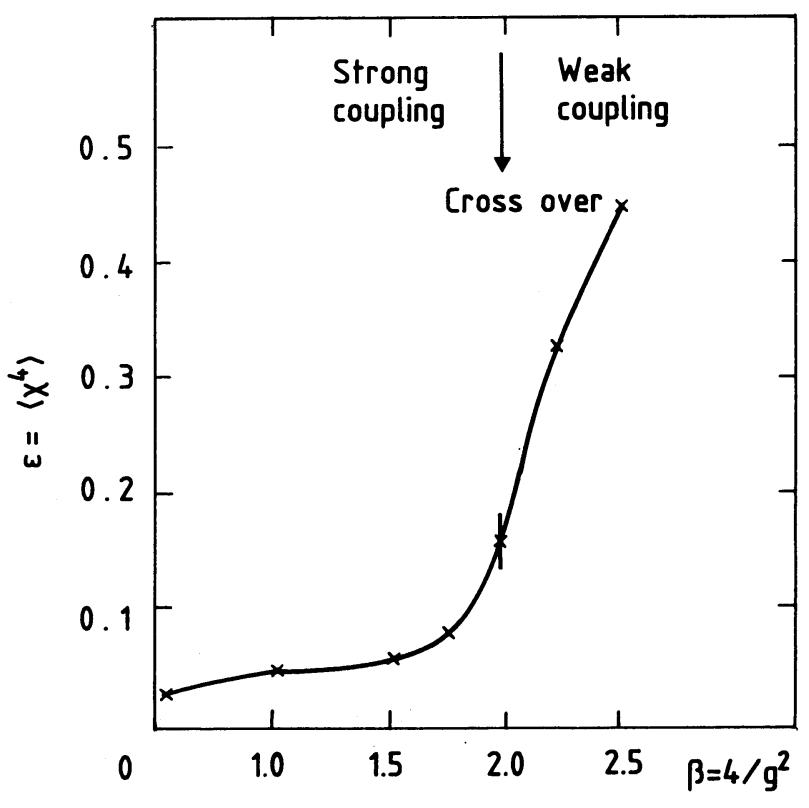

Fig. 3 Preliminary results for the dielectric constant $\langle\epsilon\rangle=\left\langle\chi^{4}\right\rangle$ in an $8^{4}$ lattice for different coupling constants $\beta=4 / \mathrm{g}^{2}$.

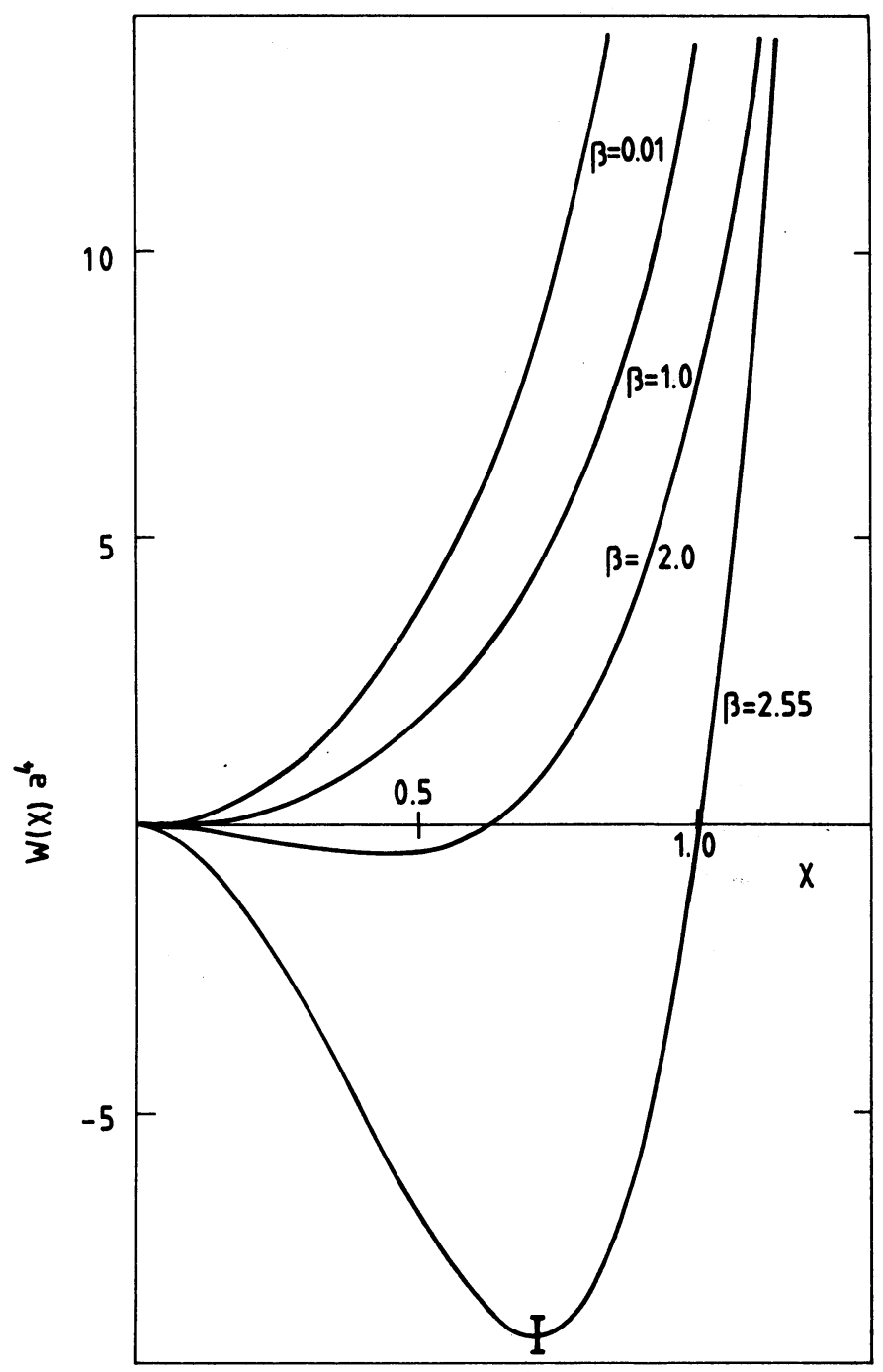

Fig. 4 Preliminary results for the $\chi$-effective action $W(\chi) a^{4}$ as a function of $\beta$. 


\section{PHENOMENOLOGY OF COLOUR DIELECTRICS}

The original starting point for a colour dielectric quark model came from the need to understand nuclear binding and the EMC effect in the framework of a bag-like model [17]. In order to understand the colour dielectric properties of nuclei, one has to introduce the coupling of the dielectric field to the quarks. In the lattice formulation the quark-gluon term is

$$
S=1 / 2 a^{3} \sum_{i, j} \bar{\psi}_{i} \gamma_{\mu} e_{\mu} U_{i j} \psi_{j}
$$

which destroys a quark at the lattice site $j$ and connects it with a link $U_{i j}$ to the site $i$ where it is created. Averaging over different links will replace $U$ by $\chi V$ or expanding $V=1+$ igaB $_{\nu} e_{\nu}$, we get, collecting terms $\propto a^{4}$,

$$
\begin{aligned}
S_{e f f}= & a^{3} \sum \bar{\psi}_{i} \gamma_{\mu} e_{\mu} \chi_{i}\left(1+i g a B_{\nu} e_{\nu}\right)\left(\psi_{i}+e_{k} \partial_{k} \psi_{i}\right) \\
& -a^{4} \sum \bar{\psi}_{i} \psi_{i} m_{q} \\
\rightarrow & \int d^{4} x\left\{\bar{\psi}(x) \chi(x)(\gamma \partial+i g \gamma B) \psi(x)-m_{a} \bar{\psi} \psi\right\}
\end{aligned}
$$

Using the covariant derivative

$$
D_{\mu}=\partial_{\mu}+\operatorname{igB}_{\mu}
$$

the total phenomenological Lagrangian has the form

$$
\mathcal{L}=\mathscr{L}(\chi)+i \chi \bar{\psi} \gamma_{\mu}\left(\partial^{u}+i B^{u}\right) \psi-\bar{\psi} \psi m_{q}-1 / 4 g^{2} \chi^{4 B_{\mu \nu} B_{\mu \nu}}
$$

where

$$
\mathrm{B}_{\mu \nu}=\left(\partial_{\mu}+i g \mathrm{~B}_{\mu}\right) \mathrm{B}_{\nu}-\left(\partial_{\nu}-\mathrm{igB} \mathrm{B}_{\nu}\right) \mathrm{B}_{\mu}
$$

and $\mathscr{L}(\chi)=T-W$ is parametrized with

$$
W(\chi)=B \chi^{2}\left(A+6-2(A+4) \chi+(A+3) \chi^{2}\right)
$$

and

$$
T(\chi)=1 / 2 \sigma_{V}^{2}\left(\partial_{\mu} \chi\right)^{2} .
$$

This phenomenological form of $W(\chi)$ is chosen in accordance with our previous work [18] on the pure gluon theory, where we set $\chi=\left(\sigma_{\mathrm{v}}-\sigma\right) / \sigma_{\mathrm{v}}$. Note that the effective pure gluonic Lagrangian of Eq. (18) is similar to the Friedberg-Lee soliton model $[19,20]$ in this case. The dielectric constant is defined in the Lagrangian of Eq. (18) as $\epsilon=\chi^{4}$.

The fermion part of the colour dielectric model in Eq. (18), however, is totally different from the Friedberg-Lee model. In particular we note that the colour dielectric field leads to absolute quark confinement, since with $\langle\chi\rangle=0$ in the vacuum the quarks can no longer propagate, i.e. their effective mass becomes infinite. To demonstrate this we introduce a new effective fermion field $\widetilde{\psi}$ by rescaling the original field $\psi$

$$
\widetilde{\psi}=\sqrt{\chi} \psi
$$

which shifts the $\chi$-dependence from the kinetic term in Eq. (18) to the mass term $(\mathrm{mq} / \chi) \overline{\tilde{\psi}} \widetilde{\psi}$

As a first approximation, we consider the $B_{\mu}{ }^{\prime}$ s as perturbative gluon fields, dropping the non-linear parts of the Lagrangian. Phenomenologically we consider the $\chi$-part as sufficient to describe the $0^{++}$ glueball and associate the $\chi$-propagator with the glueball mass, i.e. $W(x)$ at $\chi=0$ can be expanded as 


$$
1 / 2\left(\partial^{2} \mathrm{~W} / \partial \chi^{2}\right)_{\chi=0}=1 / 2 \mathrm{~m}_{\mathrm{GB}}^{2} \sigma_{\mathrm{V}}^{2} \chi^{2}=\mathrm{B}(\mathrm{A}+6) \chi^{2}
$$

In an earlier paper, we have used the parameters $A=-2, \sigma_{v}=160 \mathrm{MeV}$, and $B^{1 / 4}=203 \mathrm{MeV}$, which reproduce rather well the following physical observables [18]: the transition temperature $T_{c}=160 \mathrm{MeV}$ to the gluon plasma, a glueball mass $\mathrm{m}_{\mathrm{GB}}=720 \mathrm{MeV}$, and a string tension $\sqrt{\mathrm{E}} / \ell=450 \mathrm{MeV}$. In this calculation of the nucleon we have used the same parameters $\mathrm{A}, \mathrm{B}^{1 / 4}$, and $\sigma_{\mathrm{V}}$. We note in passing that we also tried to calculate the nucleon with these parameters in the Friedberg-Lee soliton bag model [20] and always obtained nucleon masses $m_{N} \geq 2 \mathrm{GeV}$. This is related to a general problem of the original bag model which must use different bag constants $B$ for the heavy and light quark systems, typically $B^{1 / 4}(c \bar{c})=200 \mathrm{MeV}$ [21] and $B^{1 / 4}(q q q) \simeq 150 \mathrm{MeV}$ [22]. We will show how different colour dielectric fields in the interior describe both systems with the same effective Lagrangian.

The only remaining input parameter is the current quark mass on a hadronic size scale. We have argued $[17,23]$ that the average light quark mass should lie between $20 \mathrm{MeV}$ and $30 \mathrm{MeV}$, i.e. it is somewhat larger than in the estimates based at $\mathrm{Q}^{2}=1 \mathrm{GeV}^{2}$. Different parameters have been chosen by Broniowsky et al. [24] and Thomas et al. [25]. Especially in the last paper the whole region of parameter space has been systematically explored.

The equations of motion for the $\chi$-field and the rescaled $\psi$-field (now written without $\sim$ ), are derived from the effective action for $\mathrm{N}$ quarks of equal mass $\mathrm{m}_{\mathrm{q}}$

$$
\left(\vec{\alpha} \cdot \vec{p}+m_{q} / \chi\right) \psi=E \psi
$$

and

$$
-\sigma_{\mathrm{V}}^{2} \vec{\nabla}^{2} \chi+\partial \mathrm{W} / \partial \chi=\mathrm{N} \bar{\psi} \psi \mathrm{m}_{\mathrm{q}} / \chi^{2}
$$

Numerically, we solve the equations for the potential $\widetilde{W}(\chi)=W(\chi-\delta)$, which has a minimum at $\chi=\delta \simeq$ $10^{-3}$ such that $\mathrm{m}_{\mathrm{q}} / \delta \geq 20 \mathrm{GeV}$. Decreasing $\delta$ even more we do not find any variation in the results. Qualitatively, one understands the solution of the $\chi$-field in the presence of the quark fields by regarding the effective potential

$$
\mathrm{W}_{\text {eff }}(\chi)=\mathrm{W}(\chi)+\mathrm{N} \bar{\psi} \psi \mathrm{m}_{\mathrm{q}} / \chi
$$

which has a minimum away from $\chi=0$.

Approximating the single-particle densities as

$$
\bar{\psi} \psi \approx 1 / 2 \bar{\psi} \gamma_{0} \psi=1 / 2 /\left(4 \pi / 3 R^{3}\right)
$$

we estimate the scalar density $\bar{\psi} \psi=0.12 \mathrm{fm}^{-3}$ for a hadron size of $R=1 \mathrm{fm}$. Then we get for $\bar{\chi}$ as the minimum of $\mathrm{W}_{\text {eff }}(\chi)\left(\mathrm{m}_{\mathrm{q}}=20 \mathrm{MeV}, \mathrm{m}_{\mathrm{GB}}^{2} \sigma_{\mathrm{V}}^{2}=0.013 \mathrm{GeV}^{4}\right)$ :

$$
\chi(0)=\bar{\chi}=0.16
$$

In Fig. 5 we show the exact solutions of the coupled equations for $\chi(r)$. Indeed the value of $\chi$ inside the hadron is not very different from zero $[\chi(0)=0.17]$. Comparing this value with $\chi(0)=0.6$ of the $3 \overline{3}$ flux tube [18] solution, we see even less change of the vacuum value of $\chi$. The corresponding quark wave functions (Fig. 6) have a reduced small component compared to the MIT bag model, since the effective quark mass inside the bag amounts to $m_{q} / \bar{\chi} \approx 120 \mathrm{MeV}$. In effect the colour dielectric model interpolates between the constituent quark model $m_{\text {eff }} \simeq 300 \mathrm{MeV}$ and the original MIT bag model $\mathrm{m}_{\mathrm{q}}=0$.

The r.m.s. radius of the quark distribution is $1.2 \mathrm{fm}$, which is larger than the experimental r.m.s. radius of $0.83 \mathrm{fm}$. The magnetic moment of $2.66 \mu_{\mathrm{N}}\left(\mu_{\mathrm{p}}=2.79 \mu_{\mathrm{N}}\right)$ and the ratio of $\mathrm{g}_{\mathrm{A}} / \mathrm{g}_{\mathrm{V}}=1.32$ are excellent results. Since $\bar{\chi}$ inside the bag is rather small it is clear that the shape of the effective potential $W(\chi)$ near $\chi=1$ does not influence the nucleon mass. Calculations with different sets of parameters $B$ and $A$ do not change the result as long as $\mathrm{m}_{\mathrm{GB}}^{2} \sigma_{\mathrm{V}}^{2}$ is kept constant. The shape of the effective potential 


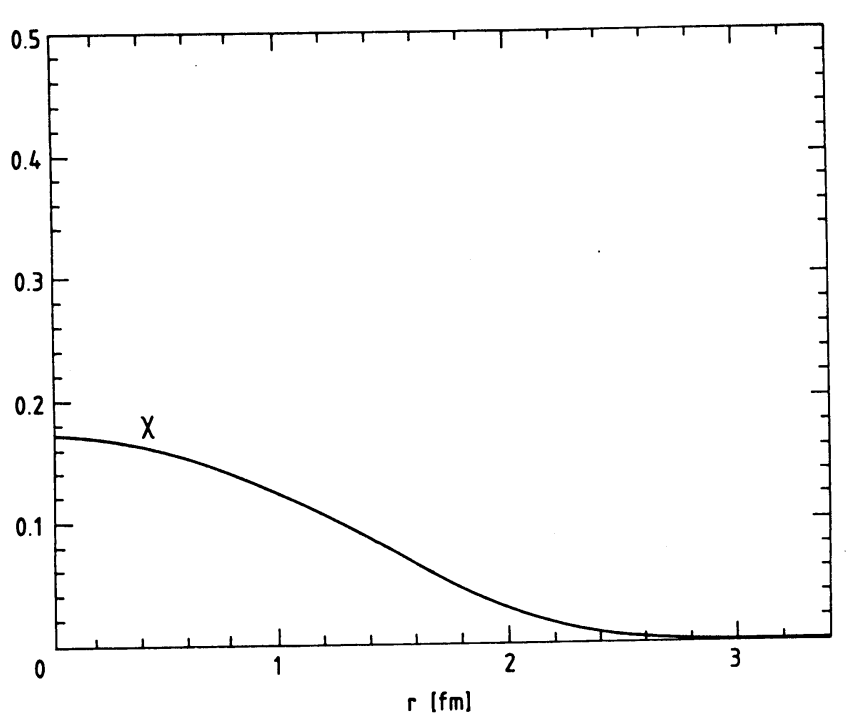

Fig. 5 The colour dielectric field $\chi(r)$ for the nucleon.

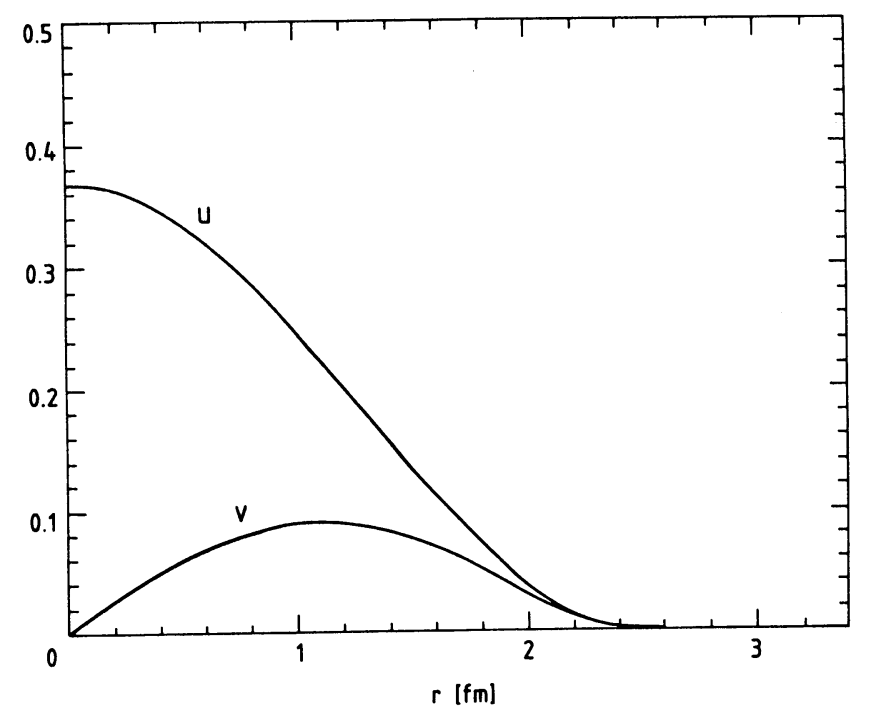

Fig. 6 The big $u(r)$ and small $u(r)$ components of the quark wave functions of the nucleon.

$W$ around $\chi=0$ determines the dynamics. This is however different for the two phase solutions of Ref. 25 , where in the interior of the bag $\chi \rightarrow 1$. It is noteworthy that the energy of the bag behaves as $\mathrm{E}_{\mathrm{bag}} \approx$ $8.5 *\left(\mathrm{~m}_{\mathrm{q}} \sigma_{\mathrm{v}} \cdot \mathrm{m}_{\mathrm{GB}}\right)^{1 / 3}$. The quark mass is given as $\mathrm{m}_{\mathrm{q}}=\mathrm{m}_{0}+$ const. $\mathrm{a}^{2}\langle\bar{\psi} \psi\rangle$, if the resolution $1 / \mathrm{a}<$ $1 \mathrm{GeV}$. In the limit of $\mathrm{m}_{0} \rightarrow 0$ the bag mass behaves as $\mathrm{E}_{\text {bag }} \propto\langle\bar{\psi} \psi\rangle^{1 / 3}$ similar to the result of QCD-sum rule calculations for the nucleon.

In the same model as used in Ref. 23 we have calculated [26] soliton matter; i.e. we have looked for quark and $\chi$-field solutions which are periodic at $r=r_{0}$, the size of a Wigner-Seitz cell. Let us recall that the nuclear matter density $\varrho$ and $r_{0}$ are related as

$$
1 /\left(4 \pi / 3 r_{0}^{3}\right)=\varrho
$$

i.e. $r_{0}$ gives the radius of the free spherical cell available to a nucleon in nuclear matter. We take $(\partial \chi / \partial r) / r_{0}$ $=0$ and $\partial\left(\psi^{\dagger} \psi\right) / \partial r=0$ as new boundary conditions at the surface of the spherical cell. The fermion boundary condition allows two solutions, which correspond in the non-relativistic limit to the symmetric and antisymmetric solutions. They give the energies at the lower-and the upper-band edge. The shape of the quark densities is rather different for both cases. The lowest energy quarks are strongly delocalized; they would belong to deeply bound 'nucleons'. At the top of the band the quarks are well localized, even compressed in comparison to their free valence quark wave function. This model is certainly very crude, but it could tell us that the quark distributions in nuclei are much less obvious than a naïve interpretation of standard nuclear physics results can teach us. Interestingly enough most existing data cover only valence nucleons such as the famous mapping out of the $3 \mathrm{~s}_{1 / 2} \mathrm{nucleons}$ in $\mathrm{Tl}$ and $\mathrm{Pb}$ with electron scattering. In a schematic model [27] similar to that described in our earlier paper [17], we have also calculated the gluon cloud corrections to the nucleon properties in nuclear matter. The non-vanishing colour dielectric field allows the quarks to leak out of their bags reducing the kinetic energy of quarks, whereby nucleons get bound, and in addition it allows a gluon cloud outside the bag. We have calculated in this more extensive model the nuclear Nolen-Schiffer anomaly. This anomaly is traced back to the deconfinement of up and down quarks in the nuclear environment which is different owing to their different masses. A crystal of nucleons (soliton matter) does not, of course, correspond to our understanding of nuclei as a liquid or a gas of nucleons. Therefore we have investigated [28] what kind of structures develop when we allow a random distribution of the colour dielectric field with two values $\chi_{\text {in }}$ and $\chi_{\text {out }}$ characterizing the values of $\chi$ inside and outside the nucleons. Such a problem is analogous to percolation studies of electrical conductivity in amorphous materials. We have used such a model before to study the interaction radius in $3 \mathrm{He}$ [29]. It has now been applied by many others to nuclei and deep inelastic scattering. Summarizing the results of this work, we show in Fig. 7 how the 


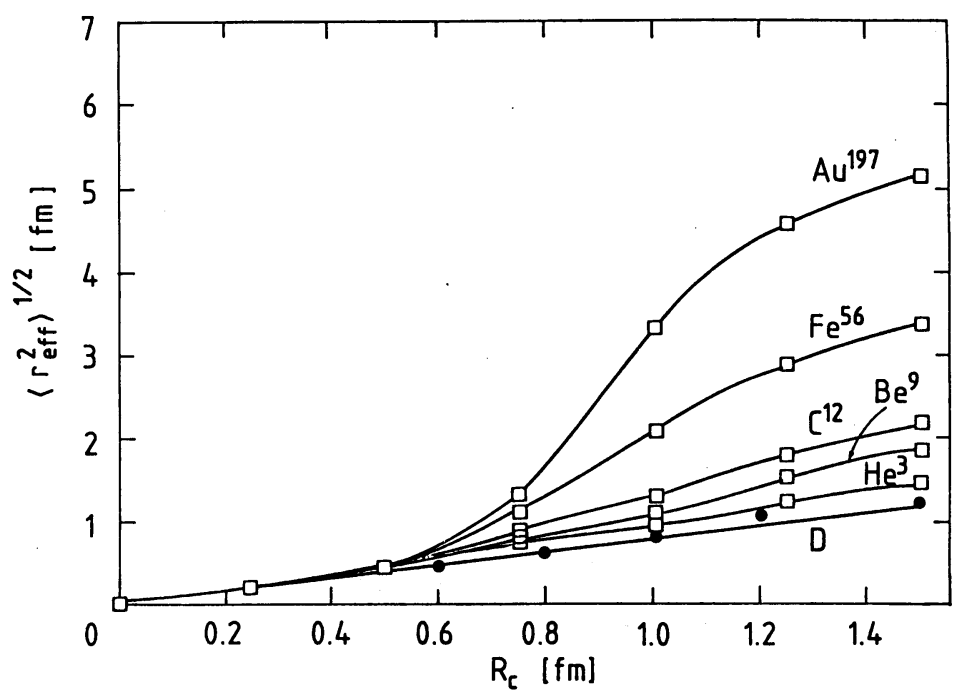

Fig. 7 The effective confinement radius $\left\langle r_{\text {eff }}^{2}\right)^{1 / 2}$ in nuclei as a function of the hard core size of the nucleon $\mathbf{R}_{\mathrm{c}}$.

effective confinement zone $\left\langle r_{\text {eff }}^{2}\right\rangle$ increases with the hard-core nucleon radius $R_{c}$, inside which $\chi=\chi_{\text {in }}$. For the deuteron we see the physics of a dilute bound state, whereas for the heavier nuclei around $R_{c}=1 \mathrm{fm}$ a transition takes place from the nuclei as colour insulators to colour conductors.

To conclude, I would like to summarize the three main theoretical points of this paper:

i) Integration over high-frequency gluon configurations generates an effective colour dielectric model Lagrangian, characterized by a few coupling constants, which can be calculated numerically.

ii) This Lagrangian generates bag-like solutions for the nucleon.

iii) This Lagrangian allows a first attempt at determining quark wave functions in nuclei.

I think that progress along these lines in non-perturbative QCD will make experiments accessible to a theoretical interpretation. Here I am mostly thinking of deep inelastic scattering experiments done at the upper edge of the available energy in the scaling region with large $x$. Existing inclusive lepton-nucleus experiments in the intermediate $x$-region have given us a glimpse of quark degrees of freedom in nuclei. Nuclear binding and a softening of the valence quark distribution are connected. New experiments at small and large $x$ will illuminate the role of sea quarks, soft gluons, and the motion of quarks in nuclei. Recently [30], we have calculated the cross section $\pi^{-} A \rightarrow \mu^{+} \mu^{-}+X$ taking into account the EMC effect and parton multiple scattering in the nucleus. In the $p_{\perp}$-distributions of the lepton pair there may be an experimental possibility to measure the color dielectric constant of nuclei.

I should like to thank the organizers of this Workshop for their kind invitation. Our work on colour dielectric lattice theory [9] has greatly benefited from discussions with Guido Martinelli, who also supplied us with an SU(3) version of the block transformation program. 


\section{REFERENCES}

[1] G. Arnison et al., Phys. Lett. 158B, 494 (1985).

[2] G. 't Hooft, Nucl. Phys. B72, 461 (1974).

[3] E. Witten, Nucl. Phys. B160, 57 (1979).

[4] E. Witten, in Recent Developments of Gauge Theories (eds. G. 't Hooft et al.) (Plenum, New York, 1980).

[5] I.J.R. Aitchison and C.M. Fraser, Phys. Lett. 146B, 63 (1984).

P. Simic, The Low Energy Meson Action from QCD, Rockefeller Univ. preprint RU/85/124 (1985).

[6] G.E. Brown and M. Rho, Comments Nucl. Part. Phys. 15, 245 (1986).

[7] C. Rebbi (ed.), Lattice Gauge Theories and Monte Carlo simulations (World Scientific, Singapore, 1983).

[8] I. Barbour, N.E. Behill, E. Dagotto, F. Karsch, A. Moreo, M. Stone and H.W. Wyld, University of Illinois preprint ILL-(TH) 86/23 (1986).

[9] H.J. Pirner, J. Wroldsen and M. Ilgenfritz, Construction of an Effective Color Dielectric Lattice Action from QCD by Blocking, CERN TH-preprint.

[10] F. Wegner, J. Math. Phys. 12, 2259 (1971).

[11] K.G. Wilson, Phys. Rev. D10, 2445 (1974).

[12] M. Creutz, Phys. Rev. D21, 2308 (1980).

[13] H.B. Nielsen and A. Patkos, Nucl. Phys. B195, 137 (1982).

[14] G. Mack, Nucl. Phys. B235, 197 (1984).

[15] K.G. Wilson, in Recent Development of Gauge Theories (eds. G. 't Hooft et al.) (Plenum Press, New York, 1980).

[16] M. Falcioni, G. Martinelli, M.L. Paciello, G. Parisi and B. Taflienti, Nucl. Phys. B, 265 [FS 15] 187 (1986).

[17] G. Chanfray, O. Nachtmann and H.J. Pirner, Phys. Lett. 147B, 249 (1984).

[18] M. Rosina, A. Schuh and H.J. Pirner, Nucl. Phys. A448, 557 (1986).

[19] R. Friedberg and T.D. Lee, Phys. Rev. D15, 1694 (1977).

[20] R. Goldflam and L. Wilets, Phys. Rev. D25, 1951 (1982).

[21] J. Baacke, Y. Igarashi and G. Kasperidus, Z. Phys. C113, 119 (1982).

[22] T. DeGrand, R.L. Jaffe, K. Johnson and J. Kishis, Phys. Rev. D12, 2060 (1975).

[23] A. Schuh and H.J. Pirner, Phys. Lett. 173B, 19 (1986).

[24] W. Broniowski, M.K. Banerjee and T.D. Cohen, University of Maryland preprint PP No. 86-081, ORO-5126-273 (1986).

[25] A.G. Williams, L.R. Dodd and A.W. Thomas, Phys. Lett. B176, 158 (1986) and A Numerical Study of a Confining Colour Dielectric Soliton Model (preprint).

[26] A. Schuh and H.J. Pirner, in preparation.

[27] G. Chanfray and H.J. Pirner, Quark Confinement and Nuclear Binding, Phys. Rev. C35, 760 (1987).

[28] F. Güttner and H.J. Pirner, Nucl. Phys. A 457, 555 (1986).

[29] H.J. Pirner and J.P. Vary, Phys. Rev. Lett. 46, 1376 (1981).

[30] P. Chiappetta and H.J. Pirner, Nuclear Effects in the production of high mass Drell-Yan pairs by $\pi^{-}$W and $\pi^{-}$D interactions, CERN-TH $4646 / 87$ to be published in Nucl. Phys. B. 